\title{
A Network Pharmacology Approach to Determine the Mechanisms of Action of Shuangshen Granules in Suppressing Pancreatic Cancer Precursor Lesions
}

\section{Juling Jiang}

China Academy of Chinese Medical Sciences Guanganmen Hospital

\section{Zhenhua Zhang}

China Academy of Chinese Medical Sciences Guanganmen Hospital

\section{Yixin Yuan}

Shanxi University of Chinese Medicine

\section{Runzhi Qi}

China Academy of Chinese Medical Sciences Guanganmen Hospital

\section{Shuntai Chen}

China Academy of Chinese Medical Sciences Guanganmen Hospital

\section{Xiwen Zhang}

China Academy of Chinese Medical Sciences Guanganmen Hospital

\section{Xing Zhang}

China Academy of Chinese Medical Sciences Guanganmen Hospital

\section{Yupeng Xi}

China Academy of Chinese Medical Sciences

\section{Qiujun Guo}

China Academy of Chinese Medical Sciences Guanganmen Hospital

\section{Honggang Zheng}

China Academy of Chinese Medical Sciences Guanganmen Hospital

\section{Baojin Hua ( $\square$ huabaojinxs@126.com )}

China Academy of Chinese Medical Sciences

Rui Liu

China Academy of Chinese Medical Sciences Guanganmen Hospital

\section{Research}

Keywords: network pharmacology, pancreatic cancer precursor lesions, Shuangshen Granules, Chinese herbal medicine, mechanism

Posted Date: December 28th, 2020 
DOI: https://doi.org/10.21203/rs.3.rs-132208/v1

License: (a) (i) This work is licensed under a Creative Commons Attribution 4.0 International License. Read Full License 


\section{Abstract}

Background and purpose: Pancreatic cancer is an insidious and highly lethal disease. Recognition and treatment of pancreatic cancer precursor lesions (PCPL) are important measures and can improve patient survival rate. Shuangshen Granules (SSG) have been prescribed for use in clinical practice for more than seven years and are widely used to treat the precursor lesions of various tumours. In this study, we used network pharmacology to explore the pharmacological mechanisms through which SSG suppress PCPL. We aimed to provide a basis for further research and the development of small, molecular, natural chemical drugs.

Methods: We first searched databases and screened the bioactive components of SSG and the related targets acting on PCPL to construct a component-target network. Then, network topology analysis was used to analyse the hub target of SSG acting on PCPL. Enrichment analyses of Gene Ontology (GO) and Kyoto Encyclopedia of Genes and Genomes (KEGG) were also performed to determine the potential pathways. Finally, molecular docking simulations were carried out to investigate the interactions between PCPL-target proteins and the active components of SSG.

Results: Seven of the main components of SSG affected PCPL, with 100 key targets including 16 hub targets. In addition, GO and KEGG enrichment analysis revealed that SSG regulated 111 molecular functions, 46 cellular components, 2334 biological processes, and 144 related signalling pathways, of which 26 were closely related to PCPL. Results of molecular docking analysis showed that the PCPLrelated targets had strong binding properties with the active components of SSG, quercetin, and ginsenoside rh2, mainly TNF, IL-6, AKT1, TP53, and EGFR.

Conclusion: This study has revealed the pharmacological and molecular mechanisms through which SSG acts on PCPL. It also provides powerful evidence to support the exploration of the pharmacological mechanisms of action and clinical applications of traditional Chinese medicine.

\section{Background}

Pancreatic cancer is one of the most lethal malignancies worldwide and ranks seventh in terms of the mortality and morbidity of cancer in both sexes. Globally, there are 458,918 new cases and 432,242 deaths reported per year [1-3], meaning rates of mortality and morbidity are almost equal. This high mortality rate is largely attributed to the difficulties in diagnosis during the early stages of the disease. Most pancreatic cancer patients, of whom are often in the middle and late stages when diagnosed [4], have poor sensitivity to chemoradiotherapy and suffer the toxic side effects of chemoradiotherapy [5]. Therefore, early detection and treatment of the cancer before it becomes invasive is particularly important in cases where a complete cure is unlikely.

Precursor lesions form as part of the process of carcinogenesis and represent a very common, unstable stage in the development and progression of cancer. They usually form as non-invasive lesions [6]. However, as they progress and are continuously stimulated by external factors, they can transform into 
invasive or metastatic carcinoma through multiple pathways. Precursor lesions are different from cancer in that they are reversible. If they can be detected early and their development stopped, this can reduce the incidence and mortality of cancer [7-8]. Therefore, increasing the efforts to understand the development, molecular mechanisms and treatment of pancreatic cancer precursor lesions is essential for the prevention and treatment of pancreatic cancer.

As an important part of conventional medicine, traditional Chinese medicine (TCM) has been used clinically for more than several thousand years. It has several benefits such as its satisfactory curative effects, and its low prevalence of side effects and cost, and has been gradually accepted by the public both in China and internationally [9-13]. Studies have shown that Chinese herbal compounds can regulate cell apoptosis via multiple pathways [14], can reduce the angiogenesis response of rats with precursor lesions, alleviate microvascular abnormalities [15] and regulate blood flow disorders which can delay, block, or reverse the development of precursor lesions [16]. This demonstrates the benefits of TCM and highlights the great potential of Chinese herbal compounds for the treatment and prevention of diseases.

The patented Chinese prescription "Shuangshen granules" (SSG; Radix Notoginseng, Cordyceps and Radix Panacis quinquefolii, Patent Number: 201310091864.4) can be effectively used to prevent tumours and treat cancer, and represents a significant development in terms of theory, practice and innovation in the field of cancer prevention and treatment. Studies have shown that Cordyceps extract can induce apoptosis, arrest the cell cycle in the $S$ phase, and increase endoplasmic reticulum stress to inhibit the proliferation of cancer cells, thus has important 'anti-cancer' roles [17-18]. In addition, Cordycepin, a bioactive component of Cordyceps, can suppress the migration and invasion of human liver cancer cells by downregulating the expression of CXCR4 [19]. Radix Panacis quinquefolii can enhance the inhibitory effect of fluorouracil on human colon cancer cells via both paraptosis and apoptosis pathways [20]; can target multiple signalling pathways such as NF-KB, JNK, and MAPK/ERK to inhibit the growth and invasion of colon cancer cells [21]; and can decrease NHE1 expression via inhibition of the EGF-EGFR-ERK1/2-HIF-1-a pathway, thus acts as a multi-target intervention for hepatocellular carcinoma [22]. The ethanol extract of pseudo-ginseng has also been shown to inhibit spleen tumours and liver metastases [23]. Its active ingredients can inhibit the proliferation of colorectal cancer cells [24-25], and tumour growth by activating macrophages to M1 polarisation [26]. In other studies, gold nanoparticles prepared from Radix Notoginseng induced cytotoxicity, ROS, and apoptosis by stimulating intrinsic apoptotic gene expression in PANC-1 cells [27]. Furthermore, Quercetin, the active ingredient in Radix Notoginseng, was shown to reverse rat liver preneoplastic lesions induced by chemical carcinogenesis [28]. All these examples provide a theoretical basis for using TCM for the prevention and treatment of pancreatic cancer precursor lesions (PCPL).

Chinese herbal compounds have multi-component, multi-target, and multi-pathway collaboration properties. However, at present, "one-target, one-drug" models of drug discovery have become increasingly inefficient; basic research is divorced from clinical applications; does not investigate new low-cost methods to screen active ingredients and predict targets; or determine all properties and mechanistic pathways of such ingredients. Therefore, it is necessary to use new scientific and technological methods to expand our understanding of the synergistic effects of SSG on PCPL to improve treatment options. Network 
pharmacology is a new pattern of drug research that analyses the relationship between drugs, targets, metabolic pathways, and diseases [28-29]. It is regarded as a promising approach for developing TCM from a systematic and molecular perspective, and can be used to provide a holistic and systematic overview of the pharmacological effects of herbs. By constructing an interaction network of different research objects, the complex relationships between nodes in the multi-level "drug-target-disease" network are identified, which aids in the understanding of the mechanism of action of traditional Chinese medicines at the system level [30].

On this basis, we consider the precursor lesions of cancer as the entry point of the disease, and the safety and effectiveness of traditional Chinese medicine as the carrier, and using a network pharmacology approach, construct a component-target network, protein-protein interaction (PPI) network, and other interaction networks at different levels. By analysing the topological parameters of these networks, a PPI network was constructed and Kyoto Gene and Genome Encyclopedia (KEGG) signalling pathway enrichment analysis was performed on the proteins in the network (Fig. 1). In addition, the targets and important signalling pathways by which SSG acts on PCPL were predicted, and the important active components and corresponding targets were simulated to explore a reliable method for the prevention and treatment of cancer and precancerous lesions using traditional Chinese medicine.

\section{Materials And Methods}

Compounds were screened based on absorption, distribution, metabolism and excretion (ADME), prediction of compound targets, collection of PCPL disease targets, network construction and analysis, Gene Ontology (GO), and KEGG signalling pathway analysis (Fig. 1).

\subsection{Screening for active components}

The components of the three herbal medicines which make up SSG were retrieved from the Traditional Chinese Medicine Systems Pharmacology Database (TCMSP, http://tcmspw.com/tcmsp.php) [32], a pharmacology platform designed for the study of Chinese herbal medicines. ADME are the four processes that drugs undergo in vivo, are important references for drug screening and discovery and can be used as tools for evaluating the pharmacokinetics of pharmaceutical components. Oral bioavailability (OB) and drug similarity $(\mathrm{DL})$ are key parameters of $\mathrm{ADME}$. The term $\mathrm{OB}$ refers to the percentage of oral medication that enters the systemic circulation, whereas DL represents whether a compound is biologically active compared to known drugs. Components with $\mathrm{OB} \geq 30 \%$ and $\mathrm{DL} \geq 0.18$, which are the recommended criteria as set out by the TCMSP database, were considered to have good oral absorption, utilisation ability, and druggability. The candidate components which met these standards were used for analysis [33-35].

\subsection{Identification of potential drug targets}

The component targets retrieved from the TCMSP database were standardised using UniProtKB (http://www.uniprot.org/) [36]. Human species attributes were selected, and protein targets from nonhuman species and without precise information were removed. In addition, the Swiss Target Prediction (http://www.swisstargetprediction.ch/) webserver [37] was used to predict the targets of the active 
components screened by ADME based on chemical similarity. Because the targets which had a validated status in the TCMSP database were verified targets, and in the Swiss Target Prediction database the probability values precisely illustrate the probability that a bioactive molecule has a given protein as a target [49], we combined the verified targets in TCMSP with the targets predicted by Swiss Target Prediction to be $100 \%$. The results from the two databases were then merged and deduplicated to obtain drug targets. An online tool (https://www.omicshare.com/) was used to analyse the distribution of the protein targets of four Chinese herbal medicines.

\subsection{Screening for disease target genes}

PCPL-related target genes were acquired from the GeneCards (https://www.genecards.org/, updated on March 11, 2020) database [38] and the Online Mendelian Inheritance in Man (OMIM; http://omim.org/, updated on Jun. 5, 2020) database [39]. In the GeneCards and OMIM databases, "pancreatic cancer precursor lesions" were selected as search keywords. In the GeneCards database, the relevance score represents the closeness of the target gene to the disease. Target genes in the GeneCards database with a score greater than 20 were selected, and the results were combined with those from the OMIM database to obtain disease targets. In order to determine candidate targets for SSG acting on PCPL, we integrated the potential targets of SSG with the target genes of PCPL to obtain the common genes.

\subsection{Network construction and analysis}

To understand and visualise the molecular mechanisms by which SSG acts on PCPL, using the herbal medicines, active components, and target information obtained, we constructed a drug-component-target network and common target protein interaction network for SSG and PCPL using Cytoscape (http://www.cytoscape.org, Ver.3.7.1) [40]. In the graphical networks, nodes represented drugs, components, or targets, with nodes as vertices and interactions as edges. We then evaluated the importance of the active components and targets according to the topological properties of the network.

\subsection{Protein-protein interactions (PPI) network}

To examine the important biological processes, PPI were analysed with a PPI network. Common genes were directed to string11.0 (https://string- $\mathrm{db}$. org, Ver.11.0) to predict related protein interactions [41]. Proteins were represented as vertices and the selection of target protein species was limited to Homo sapiens. An interaction confidence of $>0.9$ (the highest confidence to capture the precise scope of proteins related to PCPL) was set for the edges connecting corresponding proteins, in order to determine related proteins interacting directly or indirectly with the common genes of SSG and PCPL. The network diagram was then imported into Cytoscape 3.7.1 for further processing. We used the NetworkAnalyzer plug-in software to analyse the topological properties of the network [42], such as degree centrality (DC), betweenness centrality (BC), and closeness centrality (CC) [43]. DC describes the number of neighbouring nodes connected to the respective node, and nodes with high DC were considered as hub proteins in the network. In a fully connected network, any two nodes have at least one shortest path. BC refers to the number of times these shortest paths pass through the node and is an indicator of node importance. $A$ node with a high $\mathrm{BC}$ is defined as a bottleneck protein in the network. $\mathrm{CC}$ reflects how close a node is to 
other nodes or how easy it is for a node to reach other nodes. If the distance between a node and each point in the network is very short, then this point will not be subject to other points.

\subsection{Functional and pathway enrichment analysis}

In order to reveal further information regarding the potential biological pathways that these common genes may relate to, we first used the $\mathrm{R}$ (Ver.3.6.3) package org.Hs.eg.db to transfer the gene symbols to entrezIDs for subsequent analysis. Then the ClusterProfiler R/Bioconductor package was used to find the enriched GO (https://geneontology.org) items and KEGG (https://www.genome.jp/kegg) signalling pathways [44-45]. GO enrichment analysis consists of three different categories: biological processes (BP), molecular functions (MF), and cell components (CC). A value of $p<0.05$ was used as the cut-off to identify enriched $\mathrm{GO}$ terms and the localisation of the biological and molecular functions of the proteins, which indicated the relative importance of enriched $\mathrm{GO}$ terms and pathways. We also identified KEGG signalling pathways based on a $p$ value of $p<0.01$, as shown by dot plots.

\subsection{Molecular docking of component-target interaction}

In order to clarify the molecular mechanisms of SSG, Autodock4 (Ver.4.2), which is based on the Lamarckian Genetic Algorithm (LGA) [46-47] was used for molecular docking simulation. First, the proteins performing the molecular docking were downloaded from the RCSB-PDB database (https://www.RCSB.org/) [48]. These included TNF (PDB-ID: 2AZ5), IL-6 (PDB-ID: 5FUC), AKT1 (PDB-ID: 3096), TP53 (PDB-ID :4AGO) and EGFR (PDB-ID :6JXT). AutoDock Tools (Ver.1.5.6) were used to import proteins, delete water molecules, add hydrogen, and save the complex as a pdbqt file. Quercetin (MOL000098) and ginsenoside RH2 (MOL005344), the main compounds of SSG that performed molecular docking, were selected and their MOL2 structural formulas were downloaded from the TCMSP database. The compound molecules were then imported into the AutoDock Tools 1.5.6 software, it was confirmed that their flexible keys could be rotated, then they were saved as PDBQT files as docking ligands. We then set the grid box to the maximum and ran AutoGrid to budget the affinity of the atoms. AutoDock (Ver.4.2) software was used for molecular docking, and for the analysis and processing of the results. Finally, the simulation results were visualised using PyMOL (Ver.2.2).

\section{Results}

\subsection{Chemical components of SSG}

Based on the $A D M E$ thresholds of $O B \geq 30 \%$ and $D L \geq 0.18$, we obtained 23 active compounds of the whole formula from the TCMSP database. Of those compounds, Corayceps (DCXC) contained seven bioactive components, panax notoginseng (SQ) contained 8 bioactive components and 11 components were from Panacis Quinquefolii Radix (XYS), thus accounting for $30.4 \%, 34.8 \%$ and $47.8 \%$ of the compounds, respectively. There was one common component in DCXC and SQ, and two common components in SQ and XYS (Table 1). 
Table 1.

The 23 active components of the three herbs and their parameters.

\begin{tabular}{ccccc}
\hline ID Mol ID & Component & OB & DL & Herb \\
& & (\%) & & \\
\hline
\end{tabular}

DCXC1 MOL001439

DCXC2 MOL001645

DCXC3 MOL011169

DCXC4 MOL008998

DCXC5 MOL008999

DCXC6 MOL000953

CF1 MOL000358

XYS1 MOL011394

XYS2 MOL011434

XYS3 MOL011435

PQ-2

$\begin{array}{lll}45.57 & 0.20 & \text { Corayceps } \\ 42.10 & 0.20 & \text { Corayceps } \\ 44.39 & 0.82 & \text { Corayceps } \\ 39.52 & 0.77 & \text { Corayceps } \\ 31.05 & 0.45 & \text { Corayceps } \\ 37.87 & 0.68 & \text { Corayceps } \\ 36.91 & 0.75 & \text { Corayceps } \\ 36.43 & 0.25 & \text { Panacis }\end{array}$
[[(3S,5R,8R,9R,10R,12R,13R,14R,17S)-12hydroxy-4,4,8,10,14-pentamethyl-17[(2S)-6-methyl-2-[(2S,3R,4S,5S,6R)-3,4,5trihydroxy-6-(hydroxymethyl)oxan-2yl]oxyhept-5-en-2-

$$
\begin{aligned}
& \text { yl]-2,3,5,6,7,9,11,12,13,15,16,17- } \\
& \text { dodecahydro-1H-c }
\end{aligned}
$$
polyacetylene PQ-2

$36.74 \quad 0.20$

Quinquefolii

Radix 
XYS6 MOL006774

XYS7 MOL006980

XYS8 MOL008173

XYS9 MOL008397

CF1 MOL000358

CF2 MOL005344

SQ1 MOL001494

SQ2 MOL001792

SQ3 MOL002879

SQ4 MOL000449

SQ5 MOL007475

SQ6 MOL000098

CF1 MOL000358 stigmast-7-enol

papaverine

daucosterol_qt

Daturilin

beta-sitosterol

ginsenoside rh2

Mandenol

DFV

Diop

Stigmasterol

ginsenoside $\mathrm{f} 2$

quercetin

beta-sitosterol
$37.420 .75 \quad$ Panacis

Quinquefolii

Radix

$\begin{array}{lll}64.04 & 0.38 \quad \text { Panacis }\end{array}$

Quinquefolii

Radix

$36.91 \quad 0.75 \quad$ Panacis

Quinquefolii

Radix

$\begin{array}{lll}50.37 & 0.77 \quad \text { Panacis }\end{array}$

Quinquefolii

Radix

$\begin{array}{lll}36.91 & 0.75 \quad \text { Panacis }\end{array}$

Quinquefolii

Radix

$\begin{array}{lll}36.32 & 0.56 \quad \text { Panacis }\end{array}$

Quinquefolii

Radix

$\begin{array}{lll}42.00 & 0.19 \quad \text { Panax }\end{array}$

Notoginseng

$32.760 .18 \quad$ Panax

Notoginseng

$\begin{array}{lll}43.59 & 0.39 & \text { Panax }\end{array}$

Notoginseng

$43.830 .76 \quad$ Panax

Notoginseng

$36.430 .25 \quad$ Panax

Notoginseng

$46.43 \quad 0.28 \quad$ Panax

Notoginseng

$36.910 .75 \quad$ Panax

Notoginseng 


\subsection{Compound targets for SSG}

We obtained the targets and structural information regarding the 23 bioactive components from the TCMSP database. When we combined the verified targets in TCMSP with the targets predicted by Swiss Target Prediction to be $100 \%$, this resulted in a total of 223 non-duplicate targets from seven active components: arachidonic acid, peroxyergosterol, DFV, quercetin, papaverine, beta-sitosterol, and ginsenoside rh2 (Figure 2).

\subsection{PCPL target screening}

Searching the GeneCards and OMIM databases using the keywords, provided 4270 and 492 target genes related to PCPL, respectively. After screening the genes obtained from the GeneCards database based on the relevance score, this was reduced to 621 and 492 genes. When the two disease database target genes were merged, and duplicates were deleted, this left a total of 1062 potential genes.

\subsection{Compound-target network construction and analysis}

The predicted targets of SSG were matched with the genes related to PCP, then the common targets were selected as the SSG related targets in the treatment of PCPL. A total of 100 non-duplicate targets from seven active components: arachidonic acid, peroxyergosterol, DFV, quercetin, papaverine, beta-sitosterol, and ginsenoside rh2 were obtained. Based on this, a component-target network was constructed, which was composed of 110 nodes (three herbs, seven active ingredients, and 100 protein targets) and 137 edges (Figure 3). In component-target networks, the number of edges connected to a node is represented by the "degree". The results of topological analysis showed that the average target degree was 19.571 , indicating that there were multiple components interacting with multiple targets in SSG. Among these bioactive components, four high-degree components were highly correlated with multiple PCPL targets: quercetin (MOL000098, degree $=93$ ), arachidonic acid (MOL001439, degree $=16)$, beta-sitosterol (MOL000358, degree $=12)$ and ginsenoside rh2 (MOL005344, degree $=10)$. All four compounds interacted with at least 10 targets. Of these potential protein targets, 24 were the common targets of at least two compounds, and three targets were the common targets of three or more compounds: CASP3 (degree $=4)$, BAX (degree $=3$ ) and PTGS2 (degree $=3$ ). These nodes with high degrees were considered to be the hub proteins. As some hub proteins also had high BC, this indicated that these proteins play an important hub bottleneck role in the network, which may account for the therapeutic effects of SSG on PCPL.

\subsection{PPI network construction and analysis}

We submitted 100 common targets of drugs and diseases to the string 11.0 platform (https://string$\mathrm{db}$. org/) for processing. After removing some independent nodes (CYP1A1, GSTP1, CYP19A1, ABCG2, ABCC1, GSTM1), the PPI network contained 89 nodes and 418 edges (Figure 4). When the topological 
features of PPI were analysed, the mean values of DC, BC and CC were 9.4157, 0.0156 and 0.4338 , respectively. The three targets which had parameter values greater than the mean were selected as the hub targets. In total, 16 hub targets were obtained: AKT1, TP53, JUN, MAPK1, TNF, RELA, VEGFA, SRC, IL6, PIK3R1, EGFR, CCND1, MYC, FOS, CASP8 and CASP3 (Table 2).

\section{Table 2}

The components and drugs corresponding to the hub targets.

\begin{tabular}{cccc}
\hline Mol ID & Component & Hub target & Herb \\
\hline MOL000098 & quercetin & EGFR, PIK3R1, SRC, AKT1, VEGFA, & Panax Notoginseng \\
& & FOS, TNF, JUN, IL6, CASP3, TP53, & \\
MOL001439 & arachidonic & RELA, CCND1, MAPK1, CASP3 & Corayceps \\
MOL005344 & ginsenoside & TNF, CASP3 & Panax Notoginseng, Panacis \\
mOL000358 & beta- & JUN, CASP3, CASP8 & Quinquefolii Radix \\
& sitosterol & & Panax Notoginseng, Panacis \\
& & & Quinquefolii Radix, \\
& & & Corayceps \\
\hline
\end{tabular}

\subsection{GO enrichment analysis}

The occurrence of PCPLs are related to a number of biological processes. In order to clarify the mechanism by which SSG acts on PCPL, the GO of common targets was analysed on the basis of their BP, CC and MF, using the program R. The $2491 \mathrm{GO}$ terms were significantly enriched ( $p$ value $<0.05$, q value < 0.05 ), 2334 in BP which were primarily related to the regulation of cell apoptosis, reactive oxygen species metabolism, cell proliferation, cellular response to oxidative stress, inflammatory responses, regulation of cell cycle, cell migration and angiogenesis, etc.; 46 were enriched in $\mathrm{CC}$, such as the membrane region, plasma membrane raft, cytoplasm, vesicle lumen and basal part of cell, etc.; and 111 were enriched in MF, including ubiquitinlike protein ligase binding, cytokine receptor binding, kinase regulator activity, protein phosphatase binding and growth factor receptor binding (Supplementary Table 1; Figure 5). Based on these findings, complex multipath synergetic effects may explain the benefits of SSG for PCPL.

\subsection{KEGG enrichment analysis}

Enrichment analysis performed on 100 common targets of drugs and diseases identified 140 significant enrichment pathways ( $p$ value $<0.05$, $q$ value $<0.05$; Supplementary Table 2 ). Subsequently, 26 pathways that were closely related to the disease were screened, which involved a total of 81 targets, including all of the hub targets identified previously. The 26 pathways were mainly those relating to apoptosis pathways, 
including the hsa04668: TNF signalling pathway, hsa04210: Apoptosis and hsa04215: Apoptosis-multiple species; cell proliferation and migration pathways, such as the hsa04151: PI3K-Akt signalling pathway, the hsa04010: MAPK signalling pathway, the hsa04012: ErbB signalling pathway and the hsa04370: VEGF signalling pathway; immune and inflammatory pathways, such as the hsa04620: Toll-like receptor signalling pathway, the hsa04064: NF-kappa B signalling pathway and hsa04659: Th17 cell differentiation; cell drug resistance pathways, such as hsa01521: EGFR tyrosine kinase inhibitor resistance, hsa01524: Platinum drug resistance; and cell cycle pathways, such as the hsa04115: p53 signalling pathway (Table 3 ). In the target-pathway network there were 107 nodes ( 26 signalling pathways and 81 targets) and 443 edges (Figure 6). 


\section{Table 3}

The 26 major signalling pathways.

\begin{tabular}{|c|c|c|c|c|}
\hline pathway & p_value & p.adjust & q_value & Count \\
\hline hsa05212: Pancreatic cancer & $1.14 \mathrm{E}-23$ & $3.34 \mathrm{E}-22$ & $1.12 \mathrm{E}-22$ & 21 \\
\hline hsa01521: EGFR tyrosine kinase inhibitor resistance & $1.02 \mathrm{E}-21$ & $2.00 \mathrm{E}-20$ & $6.71 \mathrm{E}-21$ & 20 \\
\hline hsa04668: TNF signalling pathway & $3.23 \mathrm{E}-21$ & $5.43 \mathrm{E}-20$ & $1.82 \mathrm{E}-20$ & 22 \\
\hline hsa04151: PI3K-Akt signalling pathway & $4.71 \mathrm{E}-20$ & $6.15 \mathrm{E}-19$ & $2.07 \mathrm{E}-19$ & 32 \\
\hline hsa04066: HIF-1 signalling pathway & $1.04 \mathrm{E}-18$ & $1.12 \mathrm{E}-17$ & $3.75 \mathrm{E}-18$ & 20 \\
\hline hsa04657: IL-17 signalling pathway & $1.20 \mathrm{E}-18$ & $1.23 \mathrm{E}-17$ & $4.13 \mathrm{E}-18$ & 19 \\
\hline hsa04010: MAPK signalling pathway & $4.24 \mathrm{E}-18$ & $3.99 \mathrm{E}-17$ & $1.34 \mathrm{E}-17$ & 28 \\
\hline hsa04210: Apoptosis & $9.99 \mathrm{E}-17$ & 8.39E-16 & $2.82 \mathrm{E}-16$ & 20 \\
\hline hsa04218: Cellular senescence & $1.72 \mathrm{E}-16$ & $1.22 \mathrm{E}-15$ & $4.11 \mathrm{E}-16$ & 21 \\
\hline hsa01524: Platinum drug resistance & $2.00 \mathrm{E}-16$ & $1.34 \mathrm{E}-15$ & $4.51 \mathrm{E}-16$ & 16 \\
\hline hsa04012: ErbB signalling pathway & $2.66 \mathrm{E}-15$ & $1.56 \mathrm{E}-14$ & $5.24 \mathrm{E}-15$ & 16 \\
\hline hsa04115: p53 signalling pathway & $4.22 \mathrm{E}-15$ & $2.42 \mathrm{E}-14$ & 8.13E-15 & 15 \\
\hline hsa04620: Toll-like receptor signalling pathway & $7.45 \mathrm{E}-14$ & $3.65 \mathrm{E}-13$ & $1.23 \mathrm{E}-13$ & 16 \\
\hline hsa04370: VEGF signalling pathway & $1.48 \mathrm{E}-13$ & $7.08 \mathrm{E}-13$ & $2.38 \mathrm{E}-13$ & 13 \\
\hline hsa04370: MicroRNAs in cancer & $1.67 \mathrm{E}-13$ & $7.86 \mathrm{E}-13$ & $2.64 \mathrm{E}-13$ & 24 \\
\hline hsa04064: NF-kappa B signalling pathway & $2.00 \mathrm{E}-11$ & $7.95 \mathrm{E}-11$ & $2.67 \mathrm{E}-11$ & 14 \\
\hline hsa05202: Transcriptional misregulation in cancer & $1.10 \mathrm{E}-10$ & $4.12 \mathrm{E}-10$ & $1.38 \mathrm{E}-10$ & 17 \\
\hline hsa04014: Ras signalling pathway & $2.63 \mathrm{E}-10$ & $9.23 \mathrm{E}-10$ & $3.10 \mathrm{E}-10$ & 18 \\
\hline hsa04659: Th17 cell differentiation & $4.10 \mathrm{E}-10$ & $1.42 \mathrm{E}-09$ & $4.76 \mathrm{E}-10$ & 13 \\
\hline hsa04630: JAK-STAT signalling pathway & $8.22 \mathrm{E}-10$ & $2.76 \mathrm{E}-09$ & $9.27 \mathrm{E}-10$ & 15 \\
\hline hsa04215: Apoptosis - multiple species & $3.01 \mathrm{E}-09$ & $9.70 \mathrm{E}-09$ & $3.26 \mathrm{E}-09$ & 8 \\
\hline hsa04110: Cell cycle & $2.80 \mathrm{E}-08$ & $8.44 \mathrm{E}-08$ & $2.83 \mathrm{E}-08$ & 12 \\
\hline hsa04150: mTOR signalling pathway & $3.35 \mathrm{E}-07$ & $8.64 \mathrm{E}-07$ & $2.90 \mathrm{E}-07$ & 12 \\
\hline hsa04062: Chemokine signalling pathway & $4.14 \mathrm{E}-07$ & $1.06 \mathrm{E}-06$ & $3.55 \mathrm{E}-07$ & 13 \\
\hline hsa04015: Rap1 signalling pathway & $1.38 \mathrm{E}-06$ & $3.47 \mathrm{E}-06$ & $1.17 \mathrm{E}-06$ & 13 \\
\hline hsa04350: TGF-beta signalling pathway & 0.005665 & 0.00951 & 0.003195 & 5 \\
\hline
\end{tabular}

3.8 Molecular docking of component-target interactions 
Molecular docking is a theoretical simulation method used to understand the interactions between protein receptors and ligands at the molecular level, and to predict their binding patterns and affinity. Several binding interactions can be identified by examining the interaction patterns of favourable energies. Due to the different binding modes, the binding affinity will have many results. A docking score of less than -5.0 . $\mathrm{kcal} / \mathrm{mol}$ indicates a good binding interaction between components and their targets [50]. All binding interactions mainly formed hydrogen bonds, and the observed binding interaction distance was less than 3.0 angstrom, indicating that the binding interaction intensity was strong or moderate (Figure 7).

In the molecular binding models for quercetin-TNF (PDB-ID:2AZ5) there were 7 binding effects; with corresponding distances of $2.0,2.0,2.1,2.2,2.2,2.6$, and $2.7 \AA$, and a binding energy of $-6.57 \mathrm{kcal} / \mathrm{mol}$ (Figure 7a). For quercetin-IL-6 (PDB-ID:5FUC), there were 3 binding effects; the corresponding distances were 2.0, 2.0, and 2.4 angstrom, and the binding energy was $-6.18 \mathrm{kcal} / \mathrm{mol}$ (Figure $7 \mathrm{~b}$ ). For quercetin-AKT1 (PDB-ID:3096) there were 7 binding effects, with corresponding distances of 2.0, 2.0, 2.2, 2.2, 2.3, and 2.8 angstrom, and a binding energy of $-6.98 \mathrm{kcal} / \mathrm{mol}$. For In quercetin-TP53 (PDB-ID:4AGO), there were 3 binding effects; the corresponding distances were $2.1,2.4$, and $2.6 \AA$, respectively, and the binding energy was $-6.56 \mathrm{kcal} / \mathrm{mol}$ (Figure 7d). For quercetin-EGFR (PDB-ID:6JXT) there were 5 binding effects; corresponding distances were 1.7, 1.8, 2.0, 2.1, and 2.5 angstrom, and the binding energy was $-7.11 \mathrm{kcal} / \mathrm{mol}$ (Figure 7e). Finally, for ginsenoside rh2-TNF(PDB-ID:2AZ5) there were two binding effects; the corresponding distances were 1.8 and 2.0 angstrom, and the binding energy was $-6.27 \mathrm{kcal} / \mathrm{mol}$ (Figure 7f).

\section{Discussion}

Pancreatic cancer is an insidious and highly lethal disease as most patients develop symptoms in the advanced stages, at which point it is largely incurable. Early prevention, diagnosis, detection and treatment are important measures to improve the survival of patients with pancreatic cancer. Recognition and treatment of PCPL are most likely to be the "Holy Grails" for the successful treatment of pancreatic cancer [51]. With thousands of years of application history, TCM has its own unique advantages as a preventive treatment option. It can be used to treat precursor lesions at all stages, and can provide an alternative or complementary strategy for the treatment of pancreatic cancer and its precursor lesions. In clinical practice based on the theory of preventive treatment of disease, SSG can be used to prevent tumours from further development. However, because of the multi-component and multi-target characteristics of TCM prescriptions, and the fact that canceration is a complex process involving multiple signalling molecules, it is difficult to determine the molecular mechanisms by which SSG acts on PCPL using conventional methods.

In this study, seven active components, 100 overlapping targets and 16 hub targets with the closest relationship between SSG and PCPL were determined. Quercetin acted on 75\% of the hub targets, demonstrating that quercetin is one of the most important components of SSG acting on PCPL, thus it may inform the development of new drugs in the future. Our results also showed that the components of SSG are involved in apoptosis, reactive oxygen metabolism, cell proliferation and migration, immune 
inflammatory responses, cell cycle, angiogenesis and other biological processes. They use ubiquitin-like protein ligase binding, cytokine receptor binding, kinase regulator activity, protein phosphatase binding and other mechanisms to act on the membrane region, cytoplasm, vesicle lumen and other parts. Signalling pathways closely related to PCPL included apoptosis pathways, cell proliferation and migration pathways, immune and inflammatory pathways, cell drug resistance pathways and cell cycle pathways. Among them, it is worth mentioning that the tumour suppressor gene, TP53 is important for regulating cell growth and proliferation. The p53 protein encoded by the TP53 gene is involved in cell cycle regulation, maintains the G2/M phase arrest and induces cell apoptosis [52]. TP53 is one of the four major drivers of PCPL, and it can be deleted or mutate in the late stages of PCPL $[51,53]$. Studies have shown that quercetin can induce cell cycle arrest and p53/Bax-dependent cell apoptosis, downregulate Akt expression, and inhibit cell differentiation and proliferation [54-58]. $\beta$-sitosterol, another important active component of SSG, has important anti-tumour effects, facilitated through its roles in cell cycle arrest and apoptosis induction. It can also mediate the p53 /NF- B/ BCRP signal axis, restoring the chemotherapeutic sensitivity of drugresistant tumour cells [59]. Chronic inflammation is an important risk factor for PCPL [60]. Studies have shown that PCPL is usually associated with high levels of immune cell infiltration, interstitial fibrosis and expression of multiple inflammatory cytokines including IL6. The inflammatory environment is an important component influencing the development of precancerous lesions. IL6 is not necessary for the formation of pancreatitis-driven PCPL, but is necessary for the maintenance and progression of lesions [61]. It can be a key factor and potential therapeutic target in all stages of pancreatic cancer development. Nuclear factor-KB (NF-KB) is an important class of nuclear transcription factors and is a key indicator of inflammatory responses. Under certain circumstances, its activation may influence the progression of chronic pancreatitis, and it can also interact with the tumour necrosis factor and VEGF, regulate PI3K-Akt expression, and mediate tumour cell proliferation and apoptosis [62]. Quercetin has been shown to inhibit NF-KB and pro-inflammatory cytokines, such as TNF- and IL-6, and reverse IL-6-induced epithelialmesenchymal transformation [63-64]. Ginsenoside Rh2 also inhibits NF-KB activation and reduces HIF-1 accumulation, and is considered a potential therapeutic candidate for chronic inflammatory diseases [65]. It also inhibits proliferation and induces apoptosis in human leukaemia cells via the TNF-signalling pathway [66]. EGFR is an important gene, which is involved in tumour proliferation, survival, metastasis and induction of angiogenesis, as well as resistance to cytotoxic chemotherapy. Studies have shown that EGFR inhibition is associated with significant suppression of tumour cell proliferation, mucin biosynthesis and multiple signalling pathways, such as AKT, Rho A, MAPK and Wnt, and has potential applications in the prevention and delay of pancreatic cancer progression [67]. Our results show that quercetin can act on EGFR, supporting results from a previous study which showed that quercetin was able to reverse EGFinduced epithelial cell transformation and invasion to the stroma through the EGFR/PI3K/Akt pathway [68].

Molecular docking results showed that the PCPL-related targets had strong binding properties with the active components of SSG, quercetin, and ginsenoside rh2, which mainly involved the TNF, IL-6, AKT1, TP53, and EGFR genes. This suggests that the active components of SSG regulated PCPL-related targets and had the potential to prevent and treat PCPL. Results from a previous experimental study support this conclusion in terms of the hub targets and signal pathway results we obtained through network pharmacology and molecular docking [68]. We suggest that the multiple compounds found in SSG can act 
on different targets of PCPL through multiple pathways. This provides a scientific basis for further research which explores the mechanisms by which SSG can be used to treat PCPL.

\section{Conclusion}

(1) SSG acts synergistically on PCPL, with multiple components, targets and pathways. This provides a scientific basis for further work to determine the mechanism by which SSG acts on PCPL.

(2) Network pharmacology revealed that the hub targets of SSG were significantly enriched in the signalling pathways of TNF, NF-KB, PI3K-Akt, EGFR and P53, indicating that SSG has effects on: tumour cell proliferation and migration, inflammatory responses, drug resistance and promoting apoptosis.

(3) According to molecular docking simulation, quercetin and ginsenoside rh2, the active components of SSG, bound well with the PCPL-related targets TNF, IL-6, AKT1, TP53, and EGFR. This suggest they are involved in cell proliferation, apoptosis, inflammation and drug resistance.

\section{Abbreviations}

TCM, Traditional Chinese medicine;

PCPL, Pancreatic cancer precursor lesions;

SSG, Shuangshen Granules;

ADME, Absorption, Distribution, Metabolism and Excretion;

GO, Gene Ontology;

KEGG, Kyoto Gene and Genome Encyclopedia;

PPI, Protein-Protein Interaction;

OB, Oral bioavailability;

DL, Drug similarity;

TCMSP, Traditional Chinese Medicines Systems Pharmacology Database;

OMIM, Online Mendelian Inheritance in Man;

DC, Degree Centrality;

BC, Betweenness Centrality;

CC, Closeness Centrality; 
DCXC, Cordyceps/Corayceps;

SQ, Panax Notoginseng;

XYS, Panacis Quinquefolii Radix;

BP, Biological Processes;

MF, Molecular Functions;

CC, Cell Components;

LGA, Lamarckian Genetic Algorithm;

\section{Declarations}

\section{Authors' contributions}

Juling Jiang wrote the first draft of the article. Rui Liu, Honggang Zheng and Baojin Hua are responsible for writing, reviewing, and editing. Zhenhua Zhang, Yixin Yuan, Xiwen Zhang, Runzhi Qi, Shuntai Chen, Qiujun Guo and Baojin Hua are responsible for managing the project and conducting the formal analysis. Xing Zhang and Yupeng Xi are responsible for data curation. Baojin Hua, Honggang Zheng, Rui Liu and Xing Zhang received the funding. All authors contributed to the design and implementation of the study.

\section{Funding}

This work was supported by the Fundamental Research Funds for the Central public welfare research institutes, project numbers ZZ13-YQ-023 and ZZ13-YQ-028; the Beijing Municipal Science and Technology Commission, project number Z181100001618006; The National Natural Science Foundation of China, project number 81774294; and the Beijing Natural Science Foundation of China, project number 7172186.

\section{Availability of data and materials}

The data and materials generated or analyzed during this study are available from the corresponding author on reasonable request.

\section{Ethics approval and consent to participate}

Not applicable.

\section{Consent for publication}

Not applicable.

\section{Conflict of Interest}


The authors declare no conflicts of interest.

\section{Footnotes}

Juling Jiang, Zhenhua Zhang and Yixin Yuan are equal first authors

Rui Liu and Baojin Hua are equal corresponding authors

\section{Publisher's Note}

Springer Nature remains neutral with regard to jurisdictional claims in published maps and institutional affiliations.

\section{Contributor Information}

Juling Jiang, Email: 1192296624@qq.com.

Zhenhua Zhang, Email: katiezhang606729@126.com.

Yixin Yuan, Email: 123012887@qq.com.

Runzhi Qi, Email: qrz1211@126.com.

Shuntai Chen, Email: qdyc31236@163.com.

Xiwen Zhang, Email: zhangxiwen606@126.com.

Xing Zhang, Email: zhangxing@bucm.edu.cn.

Yupeng Xi, Email: xyp2521@163.com.

Qiujun Guo, Email: drguoqiujun@126.com.

Honggang Zheng, Email: honggangzheng@126.com.

Baojin Hua, Email: huabaojinxs@126.com.

Rui Liu, Email: drliur@126.com.

\section{References}

[1] F. Bray, J. Ferlay, I. Soerjomataram, R.L. Siegel, L.A. Torre, A. Jemal, Global cancer statistics 2018: GLOBOCAN estimates of incidence and mortality worldwide for 36 cancers in 185 countries, C.A. Cancer J. Clin. 68(6) (2018) 394-424. doi:10.3322/caac.21492.

[2] A. McGuigan, P. Kelly, R.C. Turkington, C. Jones, H.G. Coleman, R.S. McCain, Pancreatic cancer: a review of clinical diagnosis, epidemiology, treatment and outcomes, World J. Gastroenterol. 24(43) (2018) 
[3] M. Ilic, I. Ilic, Epidemiology of pancreatic cancer, World J. Gastroenterol. 22(44) (2016) 9694-9705. doi:10.3748/wjg.v22.i44.9694.

[4] M.T. Moyer, R.R. Gaffney, Pancreatic adenocarcinoma, N. Engl. J. Med. 371(22) (2014) 2140. doi:10.1056/NEJMc1412266.

[5] S. Palacio, P.J. Hosein, I. Reis, et al, The nab-paclitaxel/gemcitabine regimen for patients with refractory advanced pancreatic adenocarcinoma, J. Gastrointest. Oncol. 9(1) (2018) 135-139. doi:10.21037/jgo.2017.10.12.

[6] F.T. Bosman, F. Carneiro, R.H. Hruban, N.D. Theise. WHO Classification of Tumours of the Digestive System (2010). IARC Press, Lyon.

[7] A.C. de Vries, N.C. van Grieken, C.W. Looman, et al, Gastric cancer risk in patients with premalignant gastric lesions: a nationwide cohort study in the Netherlands, Gastroenterology 134(4) (2008) 945-952. doi:10.1053/j.gastro.2008.01.071.

[8] A.B. Mariotto, K.R. Yabroff, Y. Shao, E.J. Feuer, M.L. Brown, Projections of the cost of cancer care in the United States: 2010-2020, J. Natl. Cancer Inst. 103(2) (2011) 117-128 doi:10.1093/jnci/djq495.

[9] H. Wang, J. Li, X. Yu, S.Y. Li, Integrated traditional Chinese and conventional medicine in treatment of severe community-acquired pneumonia: study protocol for a randomized placebo-controlled trial, Trials 19(1) (2018) 620. doi:10.1186/s13063-018-3005-9.

[10] L. Bai, J. Zhao, J. Gao, et al, Effects of Shuganjianpihuatanxingqi decoction on mild subclinical hypothyroidism: a SPIRIT compliant randomized controlled trial study protocol, Med. (Baltim.) 97(45) (2018) e13183. doi:10.1097/MD.0000000000013183.

[11] Y. Zhang, Y. Liu, R. Song, et al, Validating traditional Chinese syndrome features in varied stages of chronic gastritis malignant transformation: study protocol for a cross-sectional study, BMJ Open 8(8) (2018) e020939. doi:10.1136/bmjopen-2017-020939.

[12] Y. Zhang, Y. Liu, Y. Li, et al, Hierarchical and complex system entropy clustering analysis based validation for traditional Chinese medicine syndrome patterns of chronic atrophic gastritis, J. Altern. Complement. Med. 25(11) (2019) 1130-1139 doi:10.1089/acm.2017.0386.

[13] M. Liu, Z. Liu, Overview of clinical study on traditional Chinese medicine invigorating spleen and stomach, promoting blood circulation and remove blood stasis in treatment of chronic atrophic gastritis, Zhongguo Zhong Yao Za Zhi 37(22) (2012) 3361-3364.

[14] J. Xu, W. Shen, B. Pei, et al, Xiao Tan He Wei Decoction reverses MNNG-induced precancerous lesions of gastric carcinoma in vivo and vitro: regulation of apoptosis through NF-kB pathway, Biomed. 
Pharmacother. 108 (2018) 95-102. doi:10.1016/j.biopha.2018.09.012.

[15] J. Zeng, R. Yan, H. Pan, et al, Weipixiao attenuate early angiogenesis in rats with gastric precancerous lesions, B.M.C. Complement. Altern. Med. 18(1) (2018) 250. doi:10.1186/s12906-018-2309-3.

[16] J. Yin, J. Yi, C. Yang, et al, Weiqi decoction attenuated chronic atrophic gastritis with precancerous lesion through regulating microcirculation disturbance and HIF-1a signaling pathway, Evid. Based Complement. Alternat. Med. (2019) 2651037. doi:10.1155/2019/2651037.

[17] X. Zhao, X.H. Yu, G.Y. Zhang, et al, Aqueous Extracts of Cordyceps kyushuensis Kob Induce Apoptosis to Exert Anticancer Activity, BioMed Res. Int. 2018 (2018) 8412098 doi:10.1155/2018/8412098.

[18] H. Xie, X. Li, Y. Chen, M. Lang, Z. Shen, L. Shi, Ethanolic extract of Cordyceps cicadae exerts antitumor effect on human gastric cancer SGC-7901 cells by inducing apoptosis, cell cycle arrest and endoplasmic reticulum stress, J. Ethnopharmacol. 231 (2019) 230-240. doi:10.1016/j.jep.2018.11.028.

[19] Z. Guo, W. Chen, G. Dai, Y. Huang, Cordycepin suppresses the migration and invasion of human liver cancer cells by downregulating the expression of CXCR4, Int. J. Mol. Med. 45(1) (2020) 141-150. doi:10.3892/ijmm.2019.4391.

[20] J.Y. Wan, H. Yao, C.F. Zhang, et al, Red American ginseng enhances the effect of fluorouracil on human colon cancer cells via both paraptosis and apoptosis pathways, J. Appl. Biomed. 16(4) (2018) 311-319. doi:10.1016/j.jab.2018.05.002.

[21] J.L. Gao, G.Y. Lv, B.C. He, et al, Ginseng saponin metabolite 20(S)-protopanaxadiol inhibits tumor growth by targeting multiple cancer signaling pathways, Oncol. Rep. 30(1) (2013) 292-298. doi:10.3892/or.2013.2438.

[22] X. Li, J. Tsauo, C. Geng, H. Zhao, X. Lei, X. Li, Ginsenoside Rg3 decreases NHE1 expression via inhibiting EGF-EGFR-ERK1/2-HIF-1 a pathway in hepatocellular carcinoma: A novel antitumor mechanism, Am. J. Chin. Med. 46(8) (2018) 1915-1931. doi:10.1142/S0192415X18500969.

[23] P.F. Chen, L.M. Liu, Z. Chen, S.Y. Lin, W.X. Song, Y.F. Xu, Effects of ethanol extracts of Panax notoginseng on liver metastasis of B16 melanoma grafted in mice, Zhong Xi Yi Jie He Xue Bao 4(5) (2006) 500-503. doi:10.3736/jcim20060512.

[24] N.W. He, Y. Zhao, L. Guo, J. Shang, X.B. Yang, Antioxidant, antiproliferative, and pro-apoptotic activities of a saponin extract derived from the roots of panax notoginseng (Burk.) F.H. Chen, J. Med. Food 15(4) (2012) 350-359. doi:10.1089/jmf.2011.1801.

[25] C.Z. Wang, J.T. Xie, A. Fishbein, et al, Antiproliferative effects of different plant parts of panax notoginseng on SW480 human colorectal cancer cells, Phytother. Res. 23(1) (2009) 6-13. doi:10.1002/ptr.2383. 
[26] B. Kim, E.Y. Kim, E.J. Lee, et al, Panax notoginseng inhibits tumor growth through activating macrophage to M1 polarization, Am. J. Chin. Med. 46(6) (2018) 1369-1385 doi:10.1142/S0192415X18500726.

[27] L. Wang, J. Xu, Y. Yan, H. Liu, F. Li, Synthesis of gold nanoparticles from leaf panax notoginseng and its anticancer activity in pancreatic cancer PANC-1 cell lines, Artif. Cells Nanomed. Biotechnol. 47(1) (2019) 1216-1223. doi:10.1080/21691401.2019.1593852.

[28] G. Carrasco-Torres, H.C. Monroy-Ramírez, A.A. Martínez-Guerra, et al, Quercetin reverses rat liver preneoplastic lesions induced by Chemical Carcinogenesis, Oxid. Med. Cell. Longev. 2017 (2017) 4674918. doi:10.1155/2017/4674918.

[29] A.L. Hopkins, Network pharmacology, Nat. Biotechnol. 25(10) (2007) 1110-1111. doi:10.1038/nbt1007-1110.

[30] A.L. Hopkins, Network pharmacology: the next paradigm in drug discovery, Nat. Chem. Biol. 4(11) (2008) 682-690. doi:10.1038/nchembio.118.

[31] A.L. Hopkins, Network pharmacology: the next paradigm in drug discovery, Nat. Chem. Biol. 4(11) (2008) 682-690. doi:10.1038/nchembio.118.

[32] J. Ru, P. Li, J. Wang, et al, TCMSP: a database of systems pharmacology for drug discovery from herbal medicines, J. Cheminform. 6 (2014) 13. doi:10.1186/1758-2946-6-13

[33] X. Xu, W. Zhang, C. Huang, et al, A novel chemometric method for the prediction of human oral bioavailability, Int. J. Mol. Sci. 13(6) (2012) 6964-6982. doi:10.3390/ijms13066964.

[34] W.P. Walters, M.A. Murcko, Prediction of "drug-likeness", Adv. Drug Deliv. Rev. 54(3) (2002) 255-271. doi:10.1016/S0169-409X(02)00003-0.

[35] W. Feng, H. Ao, S. Yue, C. Peng, Systems pharmacology reveals the unique mechanism features of Shenzhu Capsule for treatment of ulcerative colitis in comparison with synthetic drugs [sci. rep. 2018 8(1) 16160. doi:10.1038/s41598-018-34509-1.

[36] UniProt Consortium, UniProt: a hub for protein information, Nucleic Acids Res. 43((database issue)) (2015) D204-D212. doi:10.1093/nar/gku989.

[37] D. Gfeller, A. Grosdidier, M. Wirth, A. Daina, O. Michielin, V. Zoete, SwissTargetPrediction: a web server for target prediction of bioactive small molecules, Nucleic Acids Res. 42(Web Server issue) (Web Server Issue) (2014) W32-W38. doi:10.1093/nar/gku293.

[38] G. Stelzer, N. Rosen, I. Plaschkes, et al, The GeneCards suite: from gene data mining to disease genome sequence analyses, Curr. Protoc. Bioinformatics 54(1) (2016) 30.1-1.30.33. doi:10.1002/cpbi.5. 
[39] J.S. Amberger, A. Hamosh, Searching Online Mendelian Inheritance in Man (OMIM): A KnowledgeBase of human genes and genetic phenotypes, Curr. Protoc. Bioinformatics 58(1) (2017) 2.1-1.2.12. doi:10.1002/cpbi.27.

[40] P. Shannon, A. Markiel, O. Ozier, et al, Cytoscape: a software environment for integrated models of biomolecular interaction networks, Genome Res. 13(11) (2003) 2498-2504. doi:10.1101/gr.1239303.

[41] D. Szklarczyk, J.H. Morris, H. Cook, et al, The STRING database in 2017: quality-controlled proteinprotein association networks, made broadly accessible, Nucleic Acids Res. 45(D1) (2017) D362-D368. doi:10.1093/nar/gkw937.

[42] Y. Assenov, F. Ramírez, S.E. Schelhorn, T. Lengauer, M. Albrecht, Computing topological parameters of biological networks, Bioinformatics 24(2) (2008) 282-284. doi:10.1093/bioinformatics/btm554.

[43] J. Liu, M. Li, X.J. Luo, B. Su, Systems-level analysis of risk genes reveals the modular nature of schizophrenia, Schizophr. Res. 201 (2018) 261-269. doi:10.1016/j.schres.2018.05.015.

[44] C. Zhang, Y. Zheng, X. Li, X. Hu, F. Qi, J. Luo, Genome-wide mutation profiling and related risk signature for prognosis of papillary renal cell carcinoma, Ann. Transl. Med. 7(18) (2019) 427. doi:10.21037/atm.2019.08.113

[45] D. Zhou, Y. Sun, Y. Jia, et al, Bioinformatics and functional analyses of key genes in smokingassociated lung adenocarcinoma, Oncol. Lett. 18(4) (2019) 3613-3622. doi:10.3892/ol.2019.10733.

[46] 0. Trott, A.J. Olson, AutoDock Vina: improving the speed and accuracy of docking with a new scoring function, efficient optimization, and multithreading, J. Comput. Chem. 31(2) (2010) 455-461. doi:10.1002/jcc.21334.

[47] A. Khezri, A. Karimi, F. Yazdian, et al, Molecular dynamic of curcumin/chitosan interaction using a computational molecular approach: emphasis on biofilm reduction, Int. J. Biol. Macromol. 114 (2018) 972-978. doi:10.1016/j.ijbiomac.2018.03.100.

[48] H.M. Berman, J. Westbrook, Z. Feng, et al, The Protein Data Bank, Nucleic Acids Res. 28(1) (2000) 235-242. doi:10.1093/nar/28.1.235.

[49] A. Daina, O. Michielin, V. Zoete, SwissTargetPrediction: updated data and new features for efficient prediction of protein targets of small molecules, Nucleic Acids Res. 47(W1) (2019) W357-W364. doi:10.1093/nar/gkz382

[50] K.Y. Hsin, S. Ghosh, H. Kitano, Combining machine learning systems and multiple docking simulation packages to improve docking prediction reliability for network pharmacology, PLOS ONE 8(12) (2013) e83922. doi:10.1371/journal.pone.0083922. 
[51] M. Singh, A. Maitra, Precursor lesions of pancreatic cancer: molecular pathology and clinical implications, Pancreatology 7(1) (2007) 9-19. doi:10.1159/000101873

[52] M. Löhr, G. Klöppel, P. Maisonneuve, A.B. Lowenfels, J. Lüttges, Frequency of K-ras mutations in pancreatic intraductal neoplasias associated with pancreatic ductal adenocarcinoma and chronic pancreatitis: a meta-analysis, Neoplasia 7(1) (2005) 17-23. doi:10.1593/neo.04445.

[53] F. Notta, M. Chan-Seng-Yue, M. Lemire, et al, A renewed model of pancreatic cancer evolution based on genomic rearrangement patterns, Nature 538(7625):378-382 (2016). doi:10.1038/nature19823.

[54] K. Bishayee, S. Ghosh, A. Mukherjee, R. Sadhukhan, J. Mondal, A.R. Khuda-Bukhsh, Quercetin induces cytochrome-c release and ROS accumulation to promote apoptosis and arrest the cell cycle in G2/M, in cervical carcinoma: signal cascade and drug-DNA interaction, Cell Prolif. 46(2) (2013) 153-163. doi:10.1111/cpr.12017.

[55] A.F. Clemente-Soto, E. Salas-Vidal, C. Milan-Pacheco, J.N. Sánchez-Carranza, O. Peralta-Zaragoza, L. González-Maya, Quercetin induces G2 phase arrest and apoptosis with the activation of p53 in an E6 expression-independent manner in HPV-positive human cervical cancer-derived cells, Mol. Med. Rep. 19(3) (2019) 2097-2106. doi:10.3892/mmr.2019.9850.

[56] T. Thangasamy, S. Sittadjody, G.C. Mitchell, et al, Quercetin abrogates chemoresistance in melanoma cells by modulating deltaNp73, B.M.C. Cancer 10 (2010) 282. doi:10.1186/1471-2407-10-282.

[57] Y.A. Salama, A. El-Karef, A.M. El Gayyar, N. Abdel-Rahman, Beyond its antioxidant properties: quercetin targets multiple signalling pathways in hepatocellular carcinoma in rats, Life Sci. 236 (2019) 116933. doi:10.1016/j.lfs.2019.116933.

[58] G. Carrasco-Torres, H.C. Monroy-Ramírez, A.A. Martínez-Guerra, et al, Quercetin reverses rat liver preneoplastic lesions induced by Chemical Carcinogenesis, Oxid. Med. Cell. Longev. 2017 (2017) 4674918. doi:10.1155/2017/4674918.

[59] Z. Wang, Y. Zhan, J. Xu, et al., $\beta$-sitosterol Reverses multidrug resistance via BCRP Suppression by Inhibiting the p53-MDM2 Interaction in Colorectal Cancer, J. Agric. Food Chem. 68(12) (2020) 3850-3858. doi:10.1021/acs.jafc.0c00107.

[60] P. Langer, P.H. Kann, V. Fendrich, et al, Five years of prospective screening of high-risk individuals from families with familial pancreatic cancer, Gut 58(10) (2009) 1410-1418. doi:10.1136/gut.2008.171611.

[61] Y. Zhang, W. Yan, M.A. Collins, et al, Interleukin-6 is required for pancreatic cancer progression by promoting MAPK signaling activation and oxidative stress resistance, Cancer Res. 73(20) (2013) 63596374. doi:10.1158/0008-5472.CAN-13-1558-T.

[62] Y. Feng, L.L. Zu, L. Zhang, MicroRNA-26b inhibits the tumor growth of human liver cancer through the PI3K/Akt and NF-KB/MMP-9/VEGF pathways, Oncol. Rep. 39(5) (2018) 2288-2296. 
doi:10.3892/or.2018.6289.

[63] S.S. Chen, A. Michael, S.A. Butler-Manuel, Advances in the treatment of ovarian cancer: a potential role of antiinflammatory phytochemicals, Discov. Med. 13(68) (2012) 7-17

[64] D. Yu, T. Ye, Y. Xiang, et al, Quercetin inhibits epithelial-mesenchymal transition, decreases invasiveness and metastasis, and reverses IL-6 induced epithelial-mesenchymal transition, expression of MMP by inhibiting STAT3 signaling in pancreatic cancer cells, OncoTargets Ther. 10 (2017) 4719-4729. doi:10.2147/OTT.S136840.

[65] L.H. Lian, Q. Jin, S.Z. Song, et al, Ginsenoside Rh2 downregulates LPS-induced NF- $к$ B activation through inhibition of TAK1 phosphorylation in RAW 264.7 murine macrophage, Evid. Based Complement. Alternat. Med. 2013 (2013) 646728. doi:10.1155/2013/646728.

[66] J. Huang, K. Peng, L. Wang, et al, Ginsenoside Rh2 inhibits proliferation and induces apoptosis in human leukemia cells via TNF-a signaling pathway, Acta Biochim. Biophys. Sin. (Shanghai) 48(8) (2016) 750-755. doi:10.1093/abbs/gmw049.

[67] A. Mohammed, N.B. Janakiram, Q. Li, et al, The epidermal growth factor receptor inhibitor gefitinib prevents the progression of pancreatic lesions to carcinoma in a conditional LSL-KrasG12D/+ transgenic mouse model, Cancer Prev. Res. Phila 3(11) (2010) 1417-1426. doi:10.1158/1940-6207.CAPR-10-0038.

[68] F.A. Bhat, G. Sharmila, S. Balakrishnan, et al, Quercetin reverses EGF-induced epithelial to mesenchymal transition and invasiveness in prostate cancer (PC-3) cell line via EGFR/PI3K/Akt pathway, J. Nutr. Biochem. 25(11) (2014) 1132-1139. doi:10.1016/j.jnutbio.2014.06.008

\section{Figures}




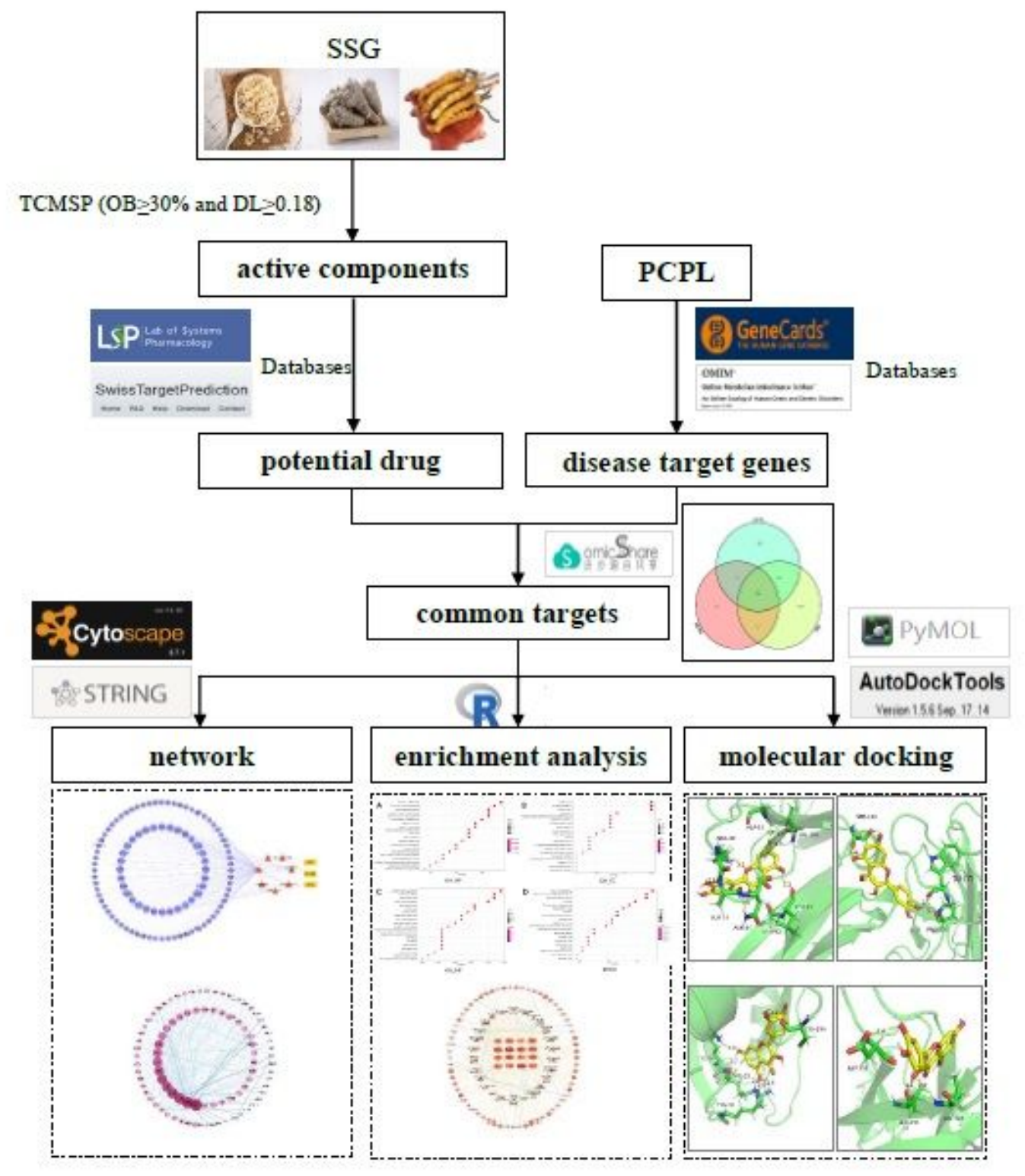

\section{Figure 1}

Flow chart of network pharmacology and molecular docking of SSG acting on PCPL. Abbreviations: SSG, Shuangshen Granules; PCPL, pancreatic cancer precursor lesions. 


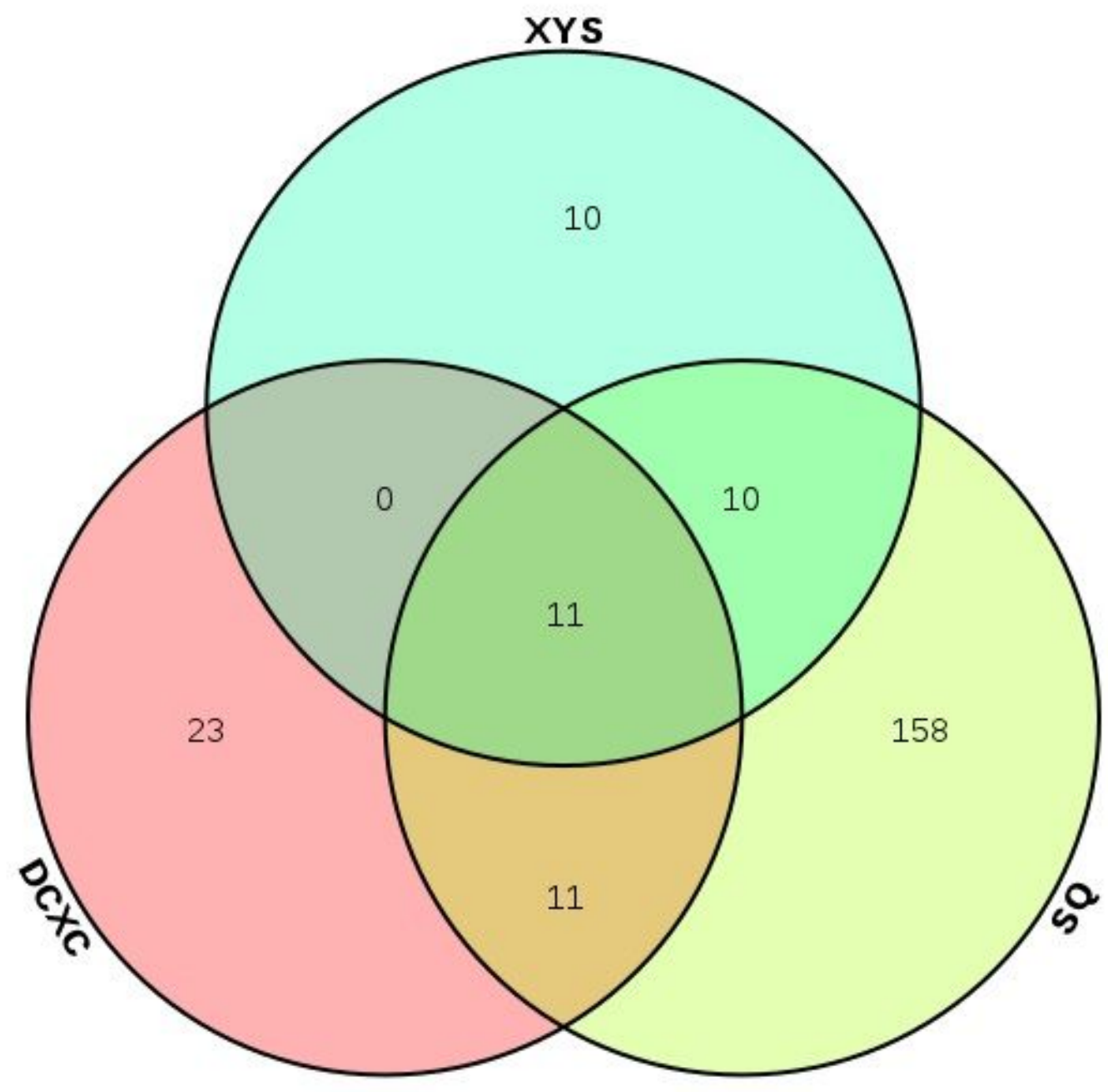

Figure 2

Venn chart of non-duplicate targets Showing the 223 non-duplicate targets corresponding to the three herbs which constitute SSG. 


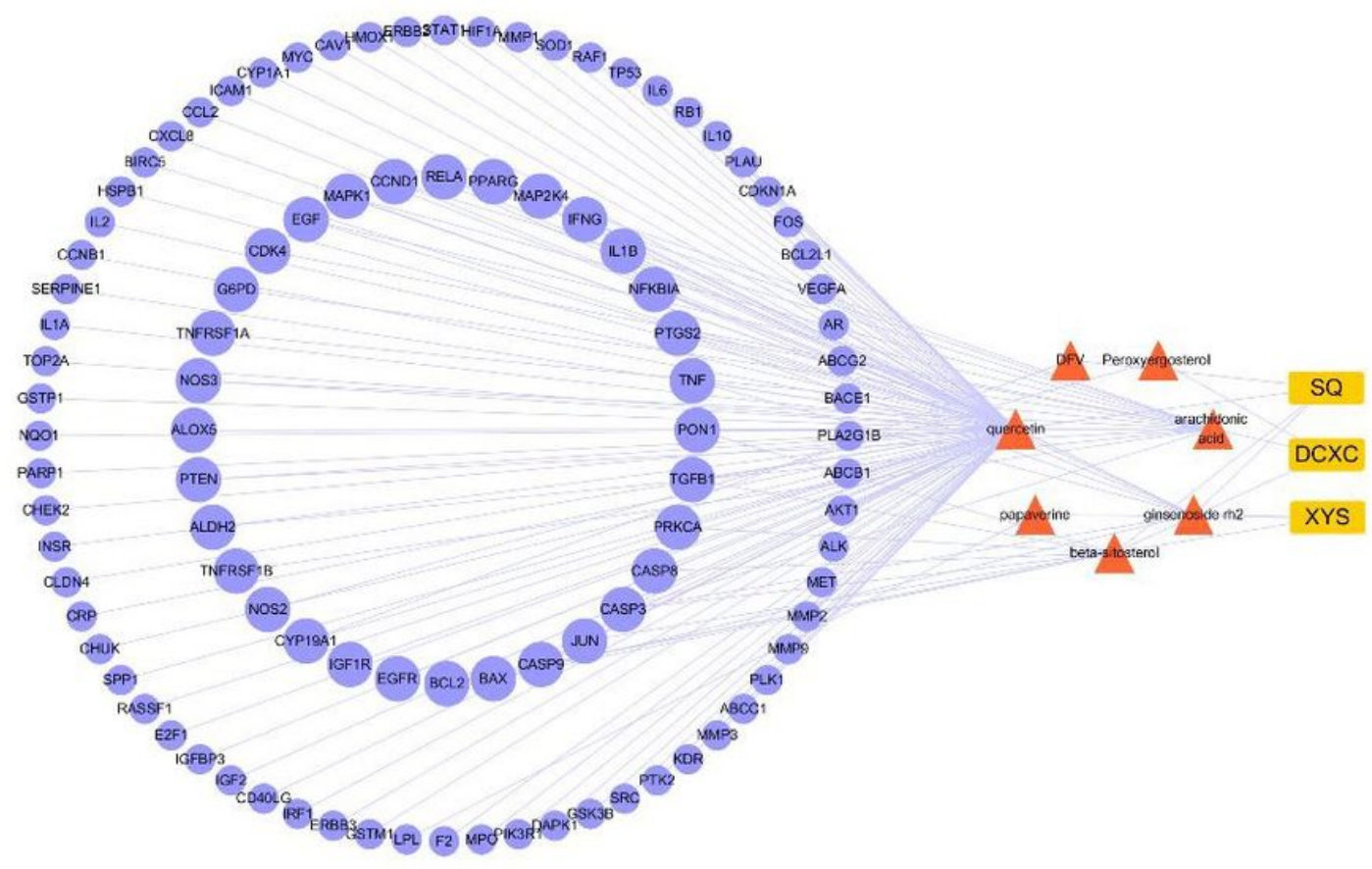

\section{Figure 3}

Compound-target network The yellow rectangles represent the three herbs which constitute SSG: Corayceps (DCXC), Panax notoginseng (SQ), and Panacis Quinquefolii Radix (XYS). The orange triangles represent the seven bioactive compounds, and the purple triangles represent the 100 overlapping targets of the drug and the disease. 


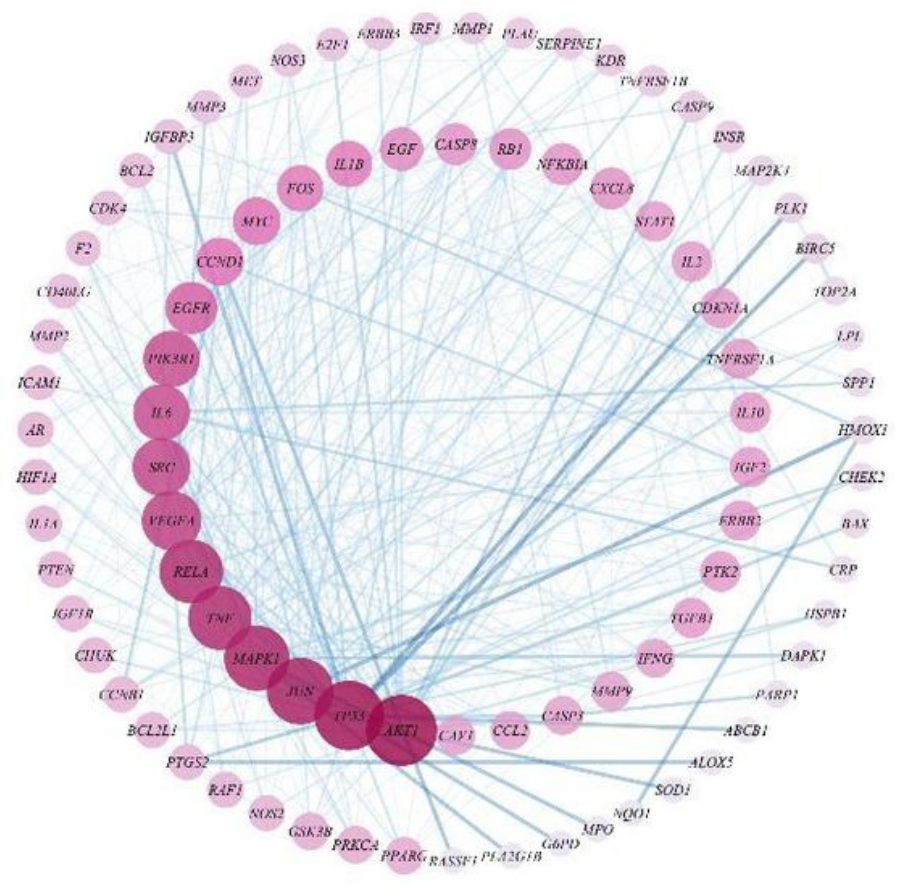

Figure 4

The SSG-PCPL PPI network The network contains 89 nodes and 418 edges. The nodes represent the common genes of drugs and diseases, and the edge represents the relationship between proteins. The pink nodes get smaller and lighter as degree value decreases. The blue edges get thinner and lighter as edge betweenness decreases. The target points with degree values of $\geqq 10$ are located in the inner circle of the network graph, and the remaining degree nodes are located in the outer circle. 

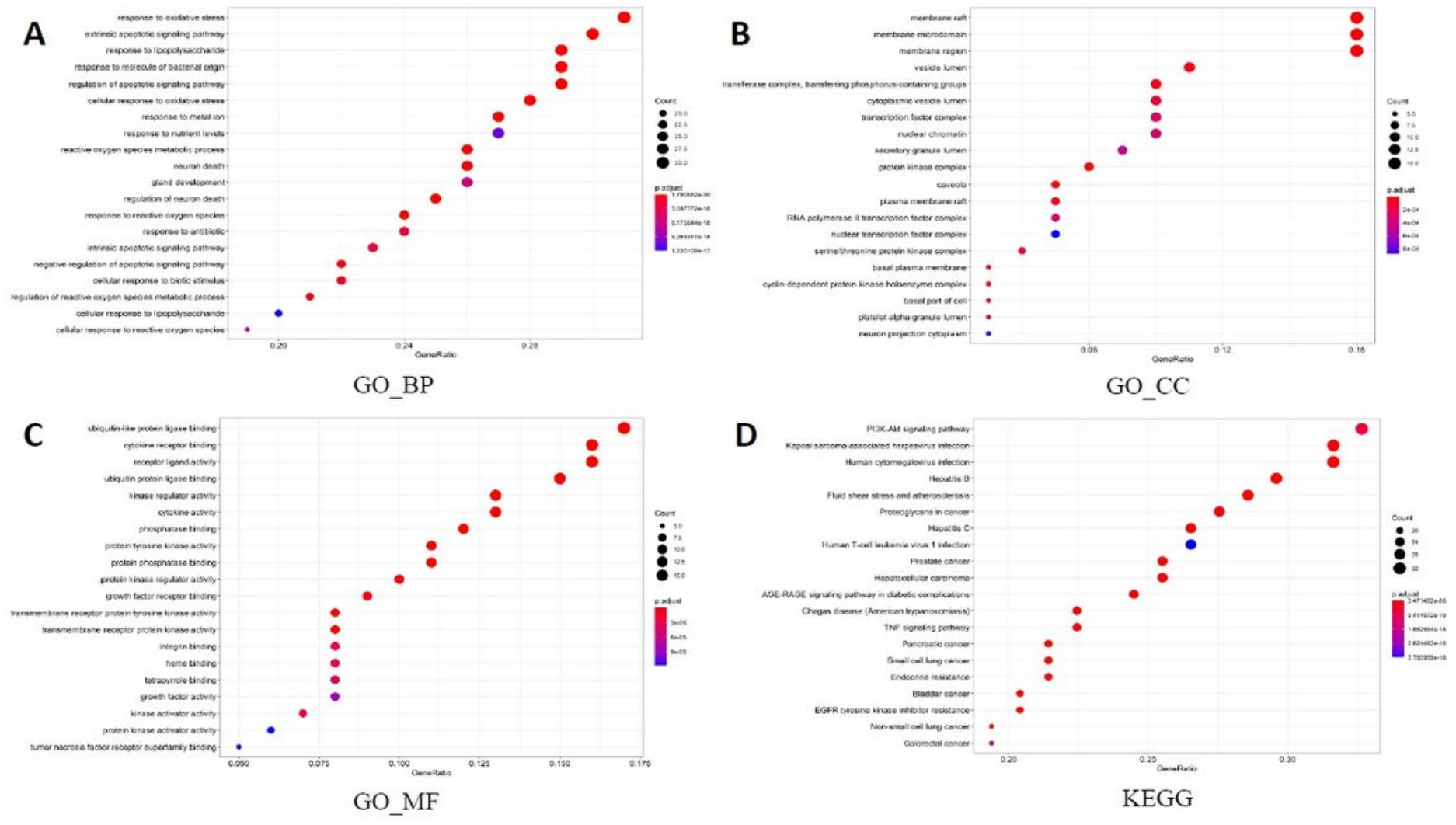

Figure 5

GO and KEGG analyses of targets. Individual plots show (A) Biological processes, (B) Cell components, (C) Molecular functions, (D) KEGG pathway analysis. The $y$-axis represents the category of significant enrichment of the targets, and the x-axis represents the enrichment scores of these terms $(p<0.01)$. Based on the adjusted $p$ values after cluster analysis, the top 20 enrichment results are shown in each figure. The colour scale represents the adjusted $p$ value, and the point size represents the number of genes in each term. Abbreviations: GO, gene ontology; KEGG, Kyoto Encyclopaedia of Genes and Genomes. 


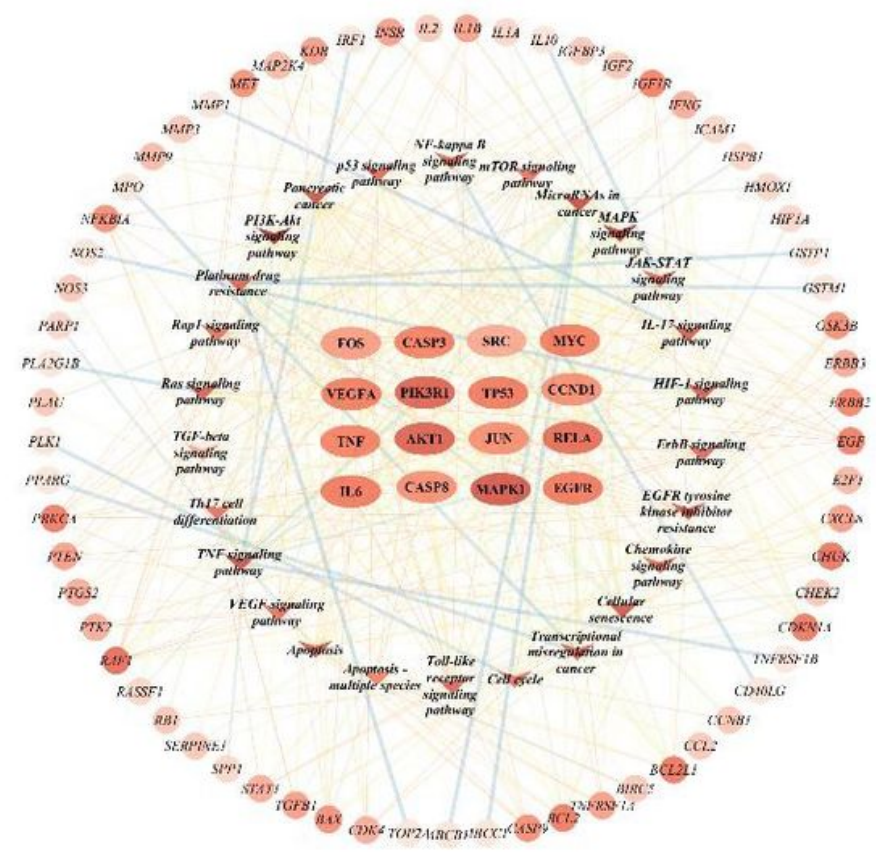

\section{Figure 6}

Target-pathway network diagram Network shows the 26 major signalling pathways. The ovals represent the core targets, the circles represent other targets on the pathway, and the inverted triangles represent the pathways. Nodes decrease in size and change colour from dark to light according to the degree value. Edges vary from thick to thin and from deep to shallow with edge betweenness. 

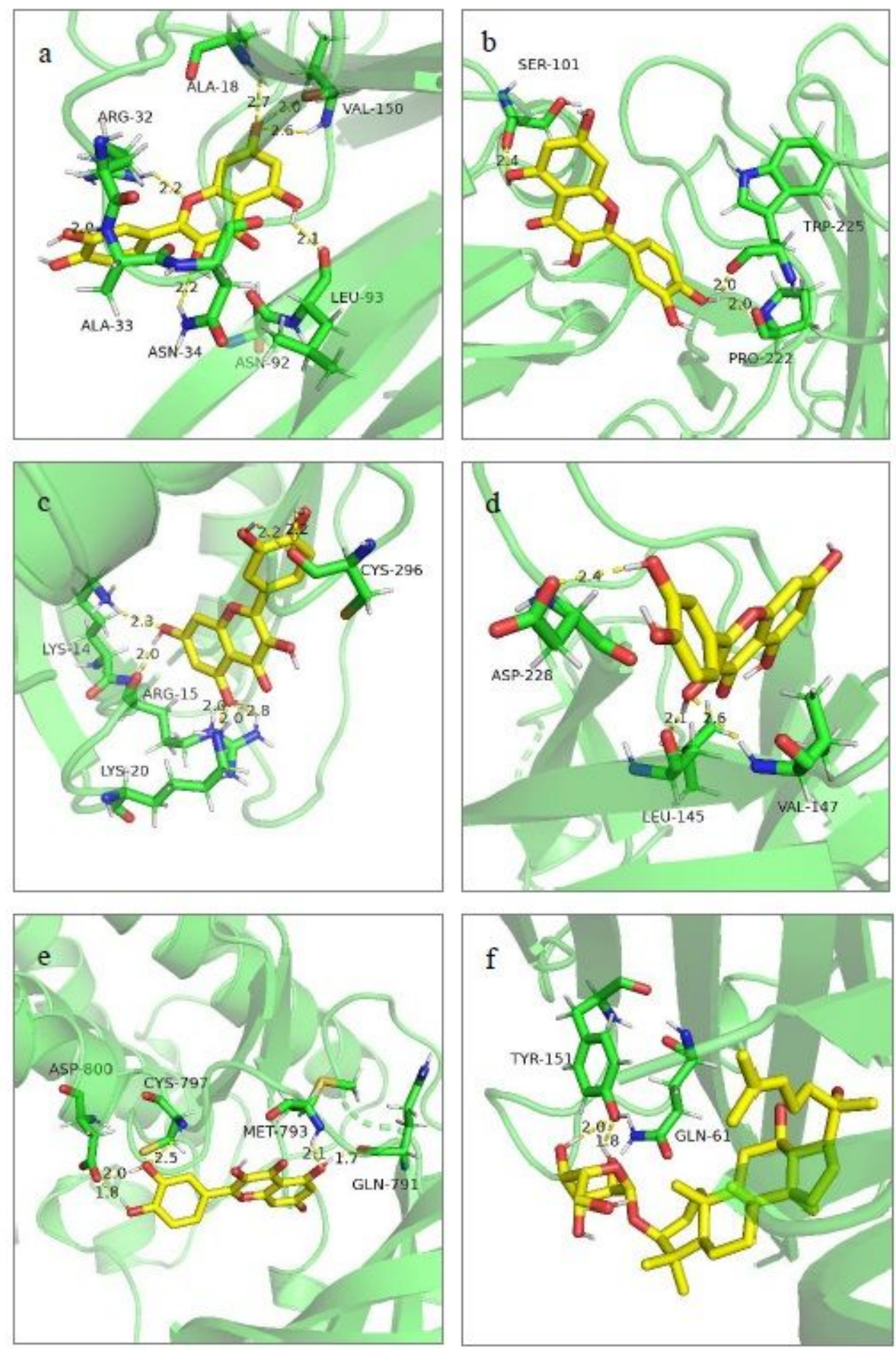

Figure 7

Molecular models for binding of active components to their predicted protein targets The sub-figures represent the molecular models for (a) quercetin-TNF (PDB-ID:2AZ5), (b) quercetin-IL-6 (PDB-ID:5FUC), (c) quercetin-AKT1 (PDB-ID:3096), (d) quercetin-TP53 (PDB-ID:4AGO), (e) quercetin-EGFR (PDB-ID:6JXT) and (f) ginsenoside rh2-TNF(PDB-ID:2AZ5). The yellow stick models represent the active components, and the 
green stick models represent residues in the binding sites. The yellow dashed lines represent hydrogen bonds; the interaction distances are indicated next to the bonds.

\section{Supplementary Files}

This is a list of supplementary files associated with this preprint. Click to download.

- SupplementaryTables.xlsx 